\title{
FOG COMPUTING IN IOT
}

\author{
R.V.Gandhi ${ }^{1}$, A.Srinivas ${ }^{2}$
}

\begin{abstract}
Fog computing is a paradigm which extends cloud computing paradigm to the edge of the network. Terms Edge Computing and Fog Computing are often used interchangeably. Similar to Cloud, Fog provides data, compute, storage, and application services to end-users. This enables new breed of applications and services.

The Internet of Things (IoT) represents a new world of information and communication technologies (ICTs) from anytime, anyplace connectivity for anyone. All Things in Internet of things or IoT are uniquely addressable and are connected using standard communication protocols. It will consist of connections that will multiply and create entirely new dynamic network of networks. In this, objects or things are made as smart so that they will become knowledgeable and their properties such as transformation, interactions will allow them to actively interact in environment.
\end{abstract}

Keywords : Fog Computing,Edge Computing,IoT.

\section{INTRODUCTION}

Fog Computing extends the Cloud Computing paradigm to the edge of the network, thus enabling a new breed of applications and services. The characteristics of the Fog are:

a) Low latency and location awareness;

b) Wide-spread geographical distribution;

c) Mobility;

d) Very large number of nodes,

e) Predominant role of wireless access,

f) Strong presence of streaming and real time applications,

g) Heterogeneity.

In this paper we discuss that the above characteristics shows that Fog the appropriate platform for a number of critical Internet of Things (IoT) services and applications, namely, Connected Vehicle, Smart Grid , Smart Cities, and, in general, Wireless Sensors and Actuators Networks (WSANs) An emerging wave of Internet deployments, most notably the Internet of Things (IoTs), requires mobility support and geo-distribution in addition to location awareness and low latency. We argue that a new platform is needed to meet these requirements; a platform we call Fog Computing [1], or, briefly, Fog, simply because the fog is a cloud close to the ground. Fog Computing enables a new breed of applications and services, and that there is a fruitful interplay between the Cloud and the Fog, particularly when it comes to data management and analytics.

\section{FOG COMPUTING AND THE INTERNET OF THINGS}

In this section we demonstrate the role the Fog plays in three scenarios of interest: Connected Vehicle, Smart Grid, and Wireless Sensor and Actuator Networks.

\subsection{CONNECTED VEHICLE (CV)}

The Connected Vehicle deployment displays a rich scenario of connectivity and interactions: cars to cars, cars to access points (Wi-Fi, 3G, LTE, roadside units [RSUs], smart traffic lights), and access points to access points. The Fog has a number of attributes that make it the ideal platform to deliver a rich menu of SCV services in infotainment, safety, traffic support, and analytics: geo-distribution (throughout cities and along roads), mobility and location awareness, low latency, heterogeneity, and support for real-time interactions. A smart traffic light system

${ }^{1}$ Asst.Professor
${ }^{2}$ Asst.Professor 
illustrates the latter. The smart traffic light node interacts locally with a number of sensors, which detect the presence of pedestrians and bikers, and measures the distance and speed of approaching vehicles. It also interacts with neighboring lights to coordinate the green traffic wave. Based on this information the smart light sends warning signals to approaching vehicles, and even modifies its own cycle to prevent accidents. Re-coordinating with neighboring STLs through the orchestration layer of the Fog follows any modification of the cycle. The data collected by the STLs is processed to do real-time analytics (changing, for instance, the timing of the cycles in response to the traffic conditions). The data from clusters of smart traffic lights is sent to the Cloud for global, longterm analytics.

\subsection{SMART GRID}

Another rich Fog use case is smart grid.

\subsection{WIRELESS SENSORS AND ACTUATORS NETWORKS}

The original Wireless Sensor Nodes (WSNs), nicknamed motes [2], were designed to operate at extremely low power to extend battery life or even to make energy harvesting feasible. Most of these WSNs involve a large number of low bandwidth, low energy, low processing power, small memory motes, operating as sources of a sink (collector), in a unidirectional fashion. Sensing the environment, simple processing, and forwarding data to the static sink are the duties of this class of sensor networks, for which the open source TinyOS2 is the de-facto standard operating system. Motes have proven useful in a variety of scenarios to collect environmental data (humidity, temperature, amount of rainfall, light intensity, etc). Energy constrained WSNs advanced in several directions: multiple sinks, mobile sinks, multiple mobile sinks, and mobile sensors were proposed in successive incarnations to meet the requirements of new applications. Yet, they fall short in applications that go beyond sensing and tracking, but require actuators to exert physical actions (open, close, move, focus, target, even carry and deploy sensors). Actuators, which can control either a system or the measurement process itself, bring new dimensions to sensor networks. The information now is not unidirectional (from the sensors to the sink), but bi-directional (sensors to sink, and controller node to actuators). In a subtler, but significant way, it becomes a closed-loop system, in which the issues of stability and potential oscillatory behavior cannot be ignored. Latency and jitter become a dominant concern in systems that require rapid response. [4] survey the contributions in the coordination of Wireless Sensor and Actuator Networks (WSANs). They point out that in one architectural choice, the WSAN consists of two networks: a wireless sensor network and a mobile ad hoc network (MANET). T. Banka et al [6] stress that emergent applications demand a higher bandwidth, collaborative sensing environment. Their experience is rooted in the CASA (Collaborative Adaptive Sensing of the Atmosphere) project. CASA [5], a multi-year, multi-partner initiative led by UMASS, deployed a network of small weather radars, integrated with a distributed processing and storage infrastructure in a closed-loop system to monitor the lower troposphere for atmospheric hazards like tornados, hailstorms, etc. Zink et al [3] provide technical details of the deployment. The characteristics of the Fog (proximity and location awareness, geo-distribution, hierarchical organization) make it the suitable platform to support both energy-constrained WSNs and WSANs.

\section{ANALYTICS, AND THE INTERPLAY BETWEEN THE FOG AND THE CLOUD}

While Fog nodes provide localization, therefore enabling low latency and context awareness, the Cloud provides global centralization. Many applications require both Fog localization, and Cloud globalization, particularly for analytics and Big Data. We touched upon this point earlier in reference to smart traffic light. Here we consider Smart Grid, which data hierarchies help illustrate further this interplay. Fog collectors at the edge ingest the data generated by grid sensors and devices. Some of this data relates to protection and control loops that require real-time processing (from milliseconds to sub seconds). This first tier of the Fog, designed for machine-to-machine (M2M) interaction, collects, process the data, and issues control commands to the actuators. It also filters the data to be consumed locally, and sends the rest to the higher tiers. The second and third tier deal with visualization and reporting (human-to machine [HMI] interactions), as well as systems and processes (M2M). The time scales of these interactions, all part of the Fog, range from seconds to minutes (real-time analytics), and even days (transactional analytics). As a result of this the Fog must support several types of storage, from ephemeral at the lowest tier to semi-permanent at the highest tier. We also note that the higher the tier, the wider the geographical coverage, and the longer the time scale. The ultimate, global coverage is provided by the Cloud, which is used as repository for data that that has a permanence of months and years, and which is the bases for business intelligence analytics. This is the typical HMI environment of reports and dashboards the display key performance indicators. 


\section{CONCLUSIONS}

We have outlined the vision and defined key characteristics of Fog Computing, a platform to deliver a rich portfolio of new services and applications at the edge of the network. The motivating examples peppered throughout the discussion range from conceptual visions to existing point solution prototypes. We envision the Fog to be a unifying platform, rich enough to deliver this new breed of emerging services and enable the development of new applications.

We welcome collaborations on the substantial body of work ahead:

1) Architecture of this massive infrastructure of compute, storage, and networking devices

2) Orchestration and resource management of the Fog nodes.

3) Innovative services and applications to be supported by the Fog.

\section{REFERENCES}

[1] F. Bonomi. Connected vehicles, the internet of things, and fog computing. VANET 2011, 2011.

[2] M. Bowman, S. K. Debray, and L. L. Peterson. Reasoning about naming systems. ACM Trans. Program. Lang. Syst., 15(5):795 \{825, November 1993.

[3] G. Forman. An extensive empirical study of feature selection metrics for text classification. J. Mach. Learn. Res., 3:1289\{1305, Mar. 2003.

[4] B. Frohlich and J. Plate. The cubic mouse: a new device for three-dimensional input. In Proceedings of the SIGCHI conference on Human factors in computing systems, CHI '00, pages 526\{531, New York, NY, USA, 2000. ACM.

[5] M. J. Sannella. Constraint satisfaction and debugging for interactive user interfaces. 2003.

[6] P. Tavel. Modeling and simulation design 2007.

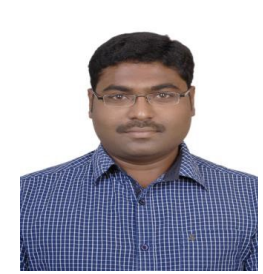

Mr.R.VENKATESWARA GANDHI, Post Graduated in Computer Science \& Engineering (M.Tech) From JNT University, Hyderabad in 2009 and Graduated in Computer Science \& engineering (B.Tech) form JNTU, Hyderabad, 2007. He is presently working as Assistant Professor in Keshav Memorial Institute of Technology,Narayanaguda,Hyderabad. He has 6+ years of Teaching Experience His Research Interests Include Network Security, Cloud Computing \& Data Warehousing and Data Mining. 